\title{
Correlación en triatlón masculino entre fases y resultado final en los JJOO de Pekin 2008
}

\section{Men's triathlon correlation between the phases and result in the Olympic Games in Beijing 2008}

\author{
Andrés B. Fernández-Revelles*, José Luis Ubago-Jiménez ${ }^{2}$, RosarioPadial-Ruz², Manuel Castro-Sánchez², \\ Virginia Viciana-Garófano ${ }^{3}$ y Pilar Puertas-Molero ${ }^{2}$ \\ 1 Dpto. Educación Física y Deportiva; Facultad de Ciencias del Deporte; Universidad de Granada (España). \\ 2 Grupo de Investigación HUM-238; Facultad de Ciencias de la Educación; Universidad de Granada (España). \\ 3 Dpto. Didáctica de la Expresión Musical, Plástica y Corporal; Facultad de Ciencias de la Educación; Universidad de Granada (España).
}

\begin{abstract}
Resumen: Introducción: El triatlón es un deporte que se compone de la combinación de tres fases de competición. La modalidad de triatlón más extendida es la olímpica, y es utilizada en los Juegos Olímpicos (JJOO), se compone de nado $1,5 \mathrm{~km}$, bicicleta $40 \mathrm{~km}$ y carrera $10 \mathrm{~km}$. Objetivos: Determinar la influencia de la duración y orden de las fases con el resultado final de la prueba en el triatlón masculino en los JJOO Pekín 2008. Método: Se analizaron los datos de 50 deportistas que finalizaron. Resultados y conclusiones: El resultado final no viene determinado ni por el orden de las fases, ni por la duración de estas, la fase menos influyente ha sido Bike con $r s=0,039 ; p=0,351$; siendo la que más tiempo ha ocupado con un $52,55 \%$; la fase más influyente ha sido la fase Run con $r s=0,991 ;$ y $p=0,000$; y $R^{2}=0,982$ y con duración $30,21 \%$.

Palabras clave: Triatlón olímpico, Juegos Olímpicos, fases, reglas de competición, reglas del juego, pruebas combinadas
\end{abstract}

Abstract: Introduction: Triathlon is a sport that is composed of the combination of three phases of competition. The most widespread triathlon is the Olympic, and is used in the Olympic Games, consists of swim, 1.5 $\mathrm{km}$, bike $40 \mathrm{~km}$ and run $10 \mathrm{~km}$. Aims: To determine the influence of the duration and order of the phases with the final result of the test in the male triathlon in the Olympic Games Beijing 2008. Method: We analyzed the data of 50 athletes who finished. Results and conclusions: The final result is not determined by the order of the phases, nor by the duration of these, the least influential phase has been Bike with $r s=.039 ; p=.351$; Being the one that has occupied the most time with $52.55 \%$; The most influential phase was the Run phase with $r s=.991$; and $p=.000$; and $R^{2}=.982$ and with duration $30.21 \%$.

Key words: Olympic triathlon, Olympic Games, phases, competition rules, game's rules, combined events

\section{Introducción}

En el inicio de este milenio se ha producido un importante aumento de la práctica deportiva en todos los niveles, a nivel de pruebas populares están teniendo mucho éxito las pruebas deportivas de media y larga distancia. Dentro de estas pruebas deportivas también proliferan aquellas que tienen lugar en diferentes espacios, incluso en entornos naturales, tales como pueden ser maratones, medios maratones, carreras de $10 \mathrm{~km}$ a pie, y además rutas en bicicleta en montaña, descensos y también en el agua travesías a nado de playas, pantanos, etc...

Junto a estas pruebas deportivas han emergido pruebas que combinan diferentes disciplinas como ocurre con el triatlón, que combina una parte nadando, la siguiente en bicicleta y la última parte carrera a pie. A estos tres segmentos o fases hay que añadirle las transiciones existentes entre las diferentes fases. Siguiendo las reglas del juego que determina

Dirección para correspondencia [Correspodence address]: Andrés B. Fernández-Revelles. Dpto. Educación Física y Deportiva; Facultad de Ciencias del Deporte; Universidad de Granada (España). E-mail: abfr@ugr.es la normativa del triatlón (ITU International Triathlon Union, 2017 b) el resultado final de la prueba se obtiene de la suma de los tiempos de las tres fases que componen la prueba más la suma de las transiciones entre ellas, resultando ganador el que haya empleado menos tiempo en la prueba y a partir de este se establece el orden de clasificación.

$\mathrm{Al}$ orden de clasificación que se establece en la llegada a meta en la última fase, o al orden de clasificación en cada una de las fases (Fernandez-Revelles, 2017, 2018; FernandezRevelles, Ubago-Jiménez, et al., 2018) le llamaremos ranking. El ranking adquiere mayor importancia cuando se establece una competición tipo campeonato o liga compuesta por diferentes pruebas de triatlón de tal forma que en función del orden en la clasificación o ranking en cada prueba se obtiene una puntuación, que se va acumulando en cada prueba, siendo el ganador de ese campeonato el que mayor puntuación obtenga.

El triatlón tiene diferentes modalidades en función sobre todo de la categoría por edad y la distancia recorrida en cada fase y en total. En todas ellas se combinan las siguientes fases por este orden nado (Swim), transición 1 (T1: Transition 1), 
bicicleta (Cyclo o Bike) transición 2 (T2: Transition 2), y carrera a pie (Run). Con la suma de los tiempos resultantes en cada fase se obtiene el tiempo total del triatlón o (Total Time). Para referirnos de forma más sencilla a cada una de las fases las llamaremos Swim, Cyclo o Bike de forma indistinta, Run, ya las dos fases de transición Transitions, y al resultado final Total Time.

La modalidad más extendida de triatlón es la modalidad olímpica, en la que las fases son Swim 1,5 km; Bike $40 \mathrm{~km}$ y Run $10 \mathrm{~km}$. Tras su aprobación para formar parte de los JJOO en 1994, por primera vez se compitió en los JJOO de Sidney 2000 tanto en categoría masculina como femenina como indica la ITU International Triathlon Union (2017b).

La competición de triatlón se rige por unas reglas del juego iguales para todos los competidores, con unas distancias anteriormente descritas fijas. Sin embargo, no existen unas pautas de tiempo para el recorrido de cada una de las fases la relación entre ellas. Puesto que los triatlones se realizan en recorridos muy diversos al aire libre, con condiciones cambiantes el tiempo empleado en cada fase por los triatletas es diferente.

El triatlón se ve enriquecido por la diversidad de deportistas y las diferencias entre las fases. Aunque las reglas del juego sean iguales para todos y están hechas para dar las mismas posibilidades de éxito a todos los triatletas, determinados mecanismos y elementos del juego, como intensidad de la prueba, adaptación del recorrido a los perfiles de los diferentes deportistas pueden influenciar a que un deportista consiga la victoria (Zapico, Benito, Diaz, Ruiz, \& Calderon, 2014).

El que se puedan realizar dentro de las reglas del juego pequeńas variaciones que no afecten a ellas, puede provocar el beneficio hacia algunos competidores por haberlo adaptado a una determinada población (Méndez-Giménez \& Fernández-Río, 2011).

Realizar un examen más profundo del triatlón como muestran los recientes estudios (Fernández-Revelles, 2017; Fernández-Revelles, Espejo-Garcés, Ubago-Jiménez, \& Chacón-Cuberos, 2018) en las pruebas de triatlón masculino de los JJOO de Sídney 2000 y Atenas 2004. En ellos se muestra el contraste existente entre la duración de las fases y la influencia en el resultado final y e incluso las discrepancias en cuanto a influencia en el resultado final entre ambas pruebas. De la misma forma no se aclara la influencia que puede tener el efecto orden de la fase en la prueba. Razones que justifican la ampliación de estudios en esta temática.

Al ser tantos y tan diferente los elementos del juego que en este tipo de competición concurren. Habría que analizar si tienen cierta relación con perfiles determinados de participante que se pueda ver beneficiado como indican (Nacke, Bateman, \& Mandryk, 2011; Rivas et al., 2015; Tondello et al., 2016).
Las pruebas de triatlón son competiciones muy exigentes y para llegar de forma adecuada a la competición son necesarios largos periodos de entrenamiento físico, técnico, psicológico, estratégico. Requiere una adecuada planificación de todos los elementos que lo componen debido a las exigencias de fuerza y fisiológicas (Bentley, Wilson, Davie, \& Zhou, 1998), potencia (Bernard et al., 2009), biomecánicas, (Le Meur et al., 2013), cognitivas, psicológicas (Jaenes Sánchez, Peñaloza Gómez, Navarrete Dueñas, \& Bohórquez GómezMillán, 2012; Leruite, Morente-Sánchez, Martos, Girela, \& Zabala, 2016). El conocimiento de la relación existente entre cada una de las fases, y cada fase con el resultado final es muy importante para tener una adecuada planificación del entrenamiento sobre todo de resistencia (Bentley, Millet, Vleck, \& McNaughton, 2002; Ramos-Campo, Martínez, Esteban, Rubio-Arias, \& Jiménez, 2016).

Existen otros elementos que, aunque también pueden ser planificados son menos predecibles y también pueden alterar el rendimiento del deportista. Son las condiciones del recorrido elementos relacionados con el entorno donde se desarrolla la prueba. De este modo hay que tener en cuenta si la prueba está ubicada en zonas tropicales o de gran humedad (Hue, 2011), o en zonas donde el frío esté muy acentuado (Dallam, Jonas, \& Miller, 2005), o que por las condiciones de la prueba el atleta pueda generar excesivo calor corporal (Kerr, Trappe, Starling, \& Trappe, 1998). Estos aspectos además van a tener una vinculación con la planificación para la prueba de la nutrición (Jeukendrup, Jentjens, \& Moseley, 2005) e hidratación (Noakes, 2007).

Una pieza importante en el desarrollo de la prueba y que también puede alterar el rendimiento de cada triatleta son algunas situaciones que puedan surgir durante la prueba. Como por el drafting en la fase Bike (Etxebarria, D’Auria, Anson, Pyne, \& Ferguson, 2014; Hausswirth \& Brisswalter, 2008; Millet \& Vleck, 2000; Peeling \& Landers, 2009). Debido a incidencias como el drafting cada triatleta conoce sus características y las posibilidades que tiene en cada fase, cuestiones que van a incidir en su estrategia durante la prueba (Abbiss $\&$ Laursen, 2008; Atkinson \& Brunskill, 2000; Hausswirth \& Brisswalter, 2008; Johnson et al., 2015; Le Meur et al., 2009)

Una situación muy común en las pruebas de triatlón es que un deportista detecte señales de agotamiento en una fase, razón por la cual en la siguiente fase ahorra energía para no caer en el agotamiento total (Bonacci, Vleck, Saunders, Blanch, \& Vicenzino, 2013). Las estrategias entre las fases o cada una de las fases va a venir condicionadas de la misma forma por la eficiencia del rendimiento en cada deportista influida por el tipo de entrenamiento y las características de este (Hausswirth \& Brisswalter, 2008; Hausswirth, Le Meur, Bieuzen, Brisswalter, \& Bernard, 2010; Le Meur et al., 2011; Peeling \& Landers, 2009).

Para poder introducir mejoras en el entrenamiento de las 
pruebas de triatlón, la planificación estratégica de este o de cualquiera de los elementos que concurren en las pruebas de triatlón. Se necesita profundizar en el conocimiento del desarrollo de la prueba, estudiar qué relaciones existen entre las fases y entre las fases y el resultado final.

\section{Objetivo}

El objetivo de este estudio es determinar en relación con el resultado final de la prueba en el triatlón masculino en los JJOO de Pekín 2008 la influencia de la duración, orden de las fases, y qué fase tiene más influencia.

\section{Material y método}

\section{Recogida de datos}

La recogida de datos para la investigación se estructuró en función de las pautas utilizadas en (Fernandez-Revelles, 2012, 2014, 2018; Fernández-Revelles, 2017; Fernandez-Revelles, Espejo-Garcés, Ubago-Jiménez, \& Chacón-Cuberos, 2018; Fernández-Revelles et al., 2018). Procedimiento que consiste en introducir en una hoja de cálculo la totalidad de os datos que coinciden con la búsqueda realizada en las bases de datos (Fernandez-Revelles, 2013; Fernandez-Revelles et al., 2009). Habiendo realizado la búsqueda con los tópicos "2008”, "men”, "triathlon”, y descargar los resultados oficiales de la competición encontrados en (ITU International Triathlon Union, 2017a).

\section{Muestra}

La muestra utilizada en el estudio es el censo completo de la prueba, eliminando los resultados de las fases de los triatletas que no finalizaron la prueba, siendo un total de 50 triatletas los la finalizaron (ITU International Triathlon Union, 2017a). Los datos en horas, minutos y segundos de las variables se transformaron a segundos utilizando una tabla de Excel y a las variables se le realizó un análisis descriptivo (tabla 1).

Tabla 1. Descriptivos de los datos recogidos

\begin{tabular}{lcccc}
\cline { 2 - 5 } & $\mathrm{N}$ & $\overline{\mathrm{X}}$ & $\sigma$ & $\sigma^{2}$ \\
\hline Swim 2008 Man & 50 & 1101,74 & 15,214 & 231,462 \\
Bike 2008 Man & 50 & 3531,48 & 20,671 & 427,275 \\
Run 2008 Man & 50 & 2030,02 & 126,781 & 16073,326 \\
Transitions 2008 Man & 50 & 56,42 & 3,137 & 9,84 \\
\hline Total Time 2008 Man & 50 & 6721,6 & 129,671 & 16814,612 \\
\hline
\end{tabular}

Como se indica anteriormente en los resultados aparecen las variables con su nomenclatura en inglés. Continuando el tra- bajo con Excel y utilizando sus fórmulas jerarquizamos las cinco variables por tiempos, para cada variable para obtener el ranking correspondiente en cada variable, procedimiento utilizado en trabajos previos (Fernandez-Revelles, 2012, 2014; Fernández-Revelles, 2017; Fernández-Revelles et al., 2018).

\section{Análisis estadístico}

Las variables se exportaron de Excel a SPSS 24 para realizar el siguiente tratamiento:

Se halló el porcentaje del tiempo utilizado en cada segmento tras realizar la suma de los tiempos de las variables.

Para determinar la relación con el resultado final de cada fase se realizó correlación de Spearman entre ranking final y rankings de las fases, (tabla 2).

Para determinar la relación entre los rankings de las fases también se utilizó correlación de Spearman, (tabla 3).

Finalmente, para ilustrar el estudio se realizaron diferentes figuras, y se halló además del parámetro $Z$ de la regresión lineal el coeficiente de determinación lineal $R^{2}$ para conocer el porcentaje de influencia.

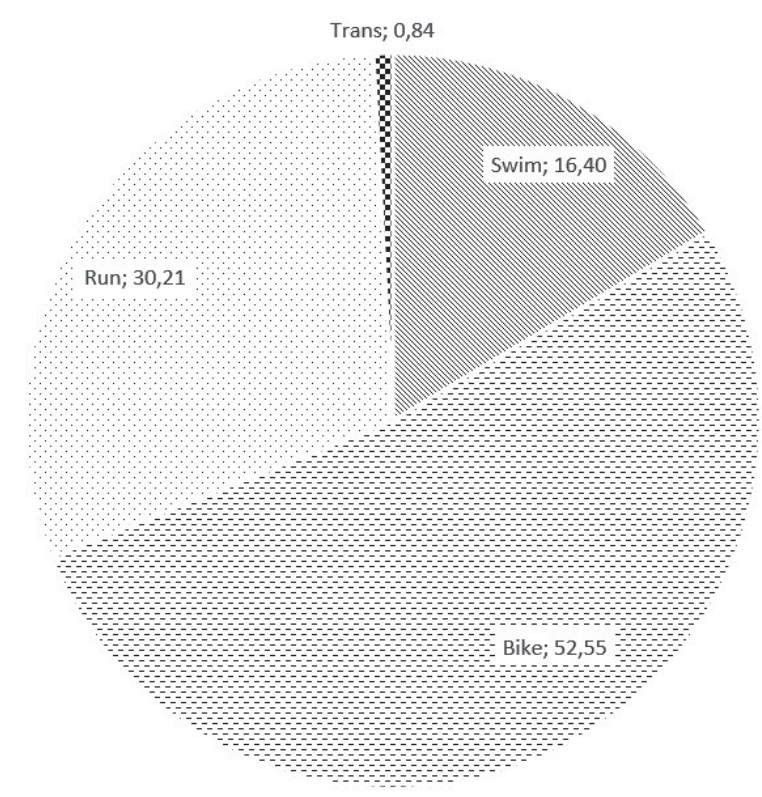

Figura 1. Porcentaje de tiempo de cada fase en relación con el tiempo total de la prueba.

\section{Resultados}

Los resultados señalan, (figura 1) como más del $52 \%$ del tiempo empleado en la prueba se ha utilizado en realizar la fase Bike, quedando en algo más del $30 \%$ Run y sólo un $16,40 \%$ Swim. 
Tabla 2. Parámetros de la correlación de Spearman entre ranking de fases y ranking final.

\begin{tabular}{lccccc}
\cline { 2 - 6 } & $\mathrm{N}$ & $\mathrm{r}_{\mathrm{s}}$ & $\mathrm{Z}$ & $\mathrm{p}$ & $\mathrm{R}^{2}$ \\
\hline Swim 2008 Men & 50 & 0,135 & 0,945 & 0,351 & 0,019 \\
Bike 2008 Men & 50 & 0,039 & 0,273 & 0,788 & 0,002 \\
Run 2008 Men & 50 & $0,991^{* *}$ & 6,937 & 0,000 & 0,982 \\
Transitions 2008 Men & 50 & $0,444^{* *}$ & 3,108 & 0,001 & 0,207 \\
${ }^{* *}$ Correlación significativa a nivel $p<0,01$ & & & \\
${ }^{*}$ Correlación significativa a nivel $p<0,05$ & & &
\end{tabular}

Tabla 3. Parámetros de la correlación de Spearman entre ranking y fases.

\begin{tabular}{lccccc}
\cline { 2 - 6 } & $\mathrm{N}$ & $\mathrm{r}_{\mathrm{s}}$ & $\mathrm{Z}$ & $\mathrm{p}$ & $\mathrm{R}^{2}$ \\
\hline Swim-Bike 2008 Men & 50 & $-0,931^{* *}$ & $-6,517$ & 0,000 & 0,851 \\
Swim-Run 2008 Men & 50 & 0,156 & 1,092 & 0,281 & 0,025 \\
$\begin{array}{l}\text { Swim-Transitions 2008 } \\
\text { Men }\end{array}$ & 50 & $-0,214$ & $-1,498$ & 0,135 & 0,045 \\
$\begin{array}{l}\text { Bike-Run 2008 Men } \\
\text { Bike-Transitions 2008 }\end{array}$ & 50 & 0,000 & 0,000 & 0,997 & $1,11 \mathrm{E}-04$ \\
$\begin{array}{l}\text { Men } \\
\text { Run-Transitions 2008 }\end{array}$ & 50 & 0,242 & 1,694 & 0,090 & 0,061 \\
Men & 50 & $0,409^{* *}$ & 2,863 & 0,003 & 0,177 \\
\hline
\end{tabular}

${ }^{* *}$ Correlación significativa a nivel $p<0,01$

*Correlación significativa a nivel $p<0,05$

Los resultados de las correlaciones de Spearman, (tabla 2), muestran que no existe correlación entre las fases Swim (figura 2) y Bike (figura 3) con el Total Time y una correlación muy alta entre la fase Run y Total Time con un $r s=0,991 ; p=$ 0,000 ; y coeficiente de determinación lineal $R^{2}=0,982$; (figura 4). Además, hay una moderada correlación entre Transitions y Total Time (figura 5) con un $r s=0,444 ; p=0,001$; y coeficiente de determinación lineal $R^{2}=0,207$.

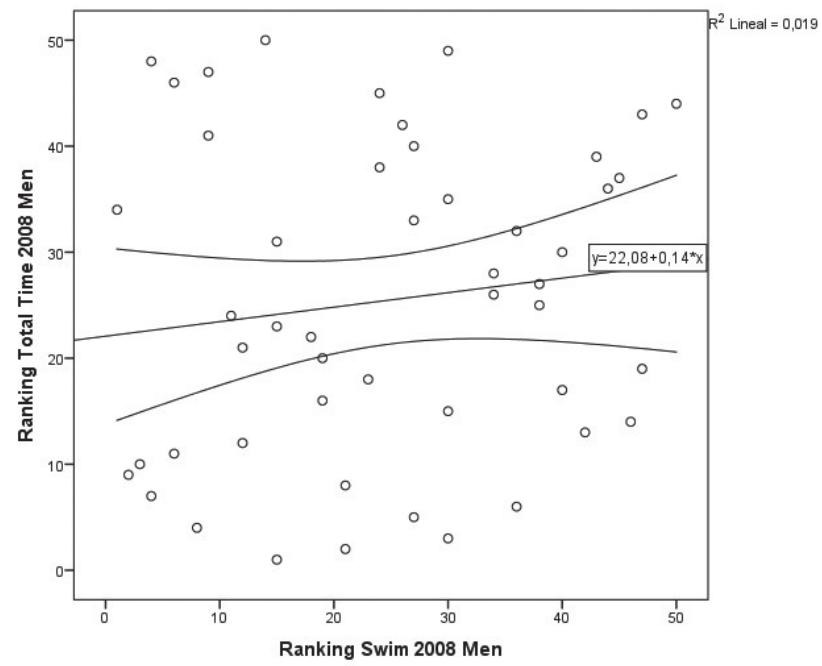

Figura 2. Relación entre ranking de la fase Swim y ranking Total Time de la prueba.
Los resultados de las correlaciones de Spearman, (tabla 3), muestran que existe correlación muy alta e inversa entre la fase Swim y Bike (figura 6) con un $r s=-0,931 ; p=0,000$; y coeficiente de determinación lineal $R^{2}=0,851$; y también existe una correlación moderada entre las fases Run y Transitions (figura 7) con un $r s=0,409 ; p=0,003$; y y coeficiente de determinación lineal $R^{2}=0,177$. No existe ningún tipo de correlación entre las otras fases.

\section{Discusión}

Los resultados muestran que el tiempo empleado en cada fase el triatlón es muy diferente, y que a más tiempo empleado en una fase no existe mayor influencia en el ranking de esa fase con el resultado final (tabla 2 y figura 8). Resultado similar al encontrado en los JJOO de Sídney 2000 donde FernándezRevelles (2017) muestra como la fase Run es la que más influencia tiene con un $85,9 \%$ y la fase Bike casi no tiene influencia con sólo un 5,1\%. En Beijing 2008 se acentúan más esas diferencias con una influencia de las fases Run de $98,24 \%$ (figura 4) y una mínima influencia de la fase Bike con 0,23\% (figura 3) aun habiendo empleado un porcentaje de tiempo en esas fases Run 30,21\% y Bike 52,55\%.

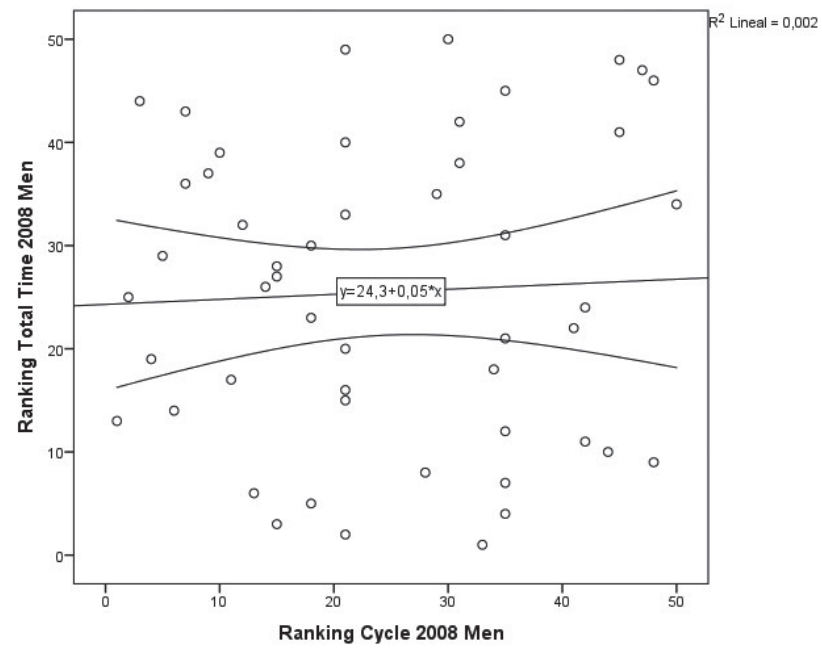

Figura 3. Relación entre ranking de la fase Cycle o Bike y ranking Total Time de la prueba.

Sin embargo, es un resultado totalmente diferente al que Fernández-Revelles et al. (2018) muestran en el triatlón de Atenas 2004 donde existe una relación directamente proporcional entre el tiempo empleado en la fase y la influencia del ranking de esa fase con el resultado final. En Atenas 2004 el porcentaje de tiempo empleado en las fases es Transitions 0,53\%; Swim $15,70 \%$; Run 29,41\%; y Bike 54,36\%; y la influencia del ranking de cada fase con el ranking del Total Time es Transition 9,97\%; Swim 17,75\%; Run 60,81\%; y Bike 76,20\%. 


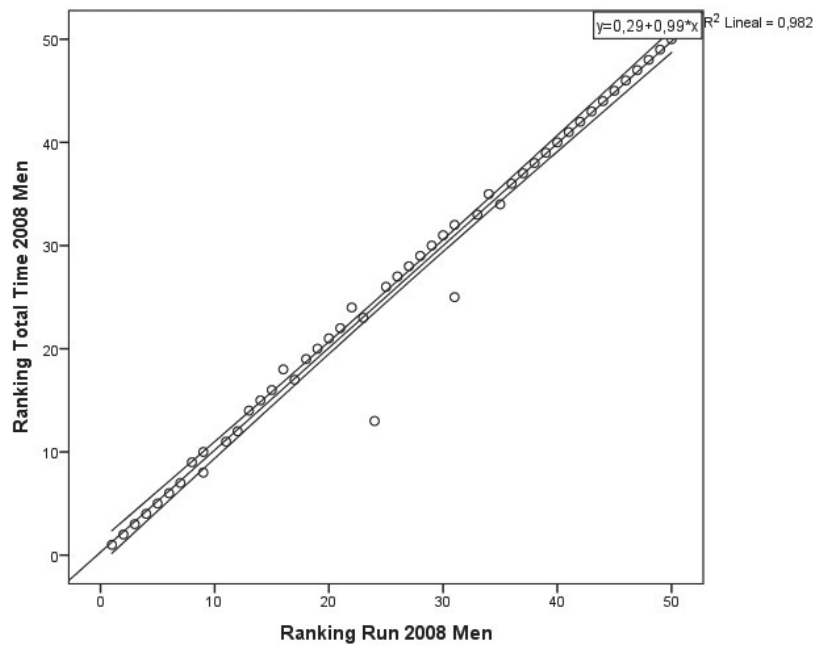

Figura 4. Relación entre ranking de la fase Run y ranking Total Time de la prueba.

El resultado del ranking de Total Time del triatlón de Beijing 2008 podría estar influenciado por el efecto orden de las fases dada la gran influencia de la fase Run con un $98,24 \%$, sin embargo, no es así puesto que el ranking de la fase Swim es $1,85 \%$; una influencia mínima, pero muy superior a la siguiente fase Bike con sólo 0,23\%. Resultados con cierta similitud al triatlón de los JJOO de Sídney 2000. Cabe destacar (figura 5) la influencia de la fase Transition con $28,19 \%$ en relación con el ranking del Total Time en Beijing 2008.

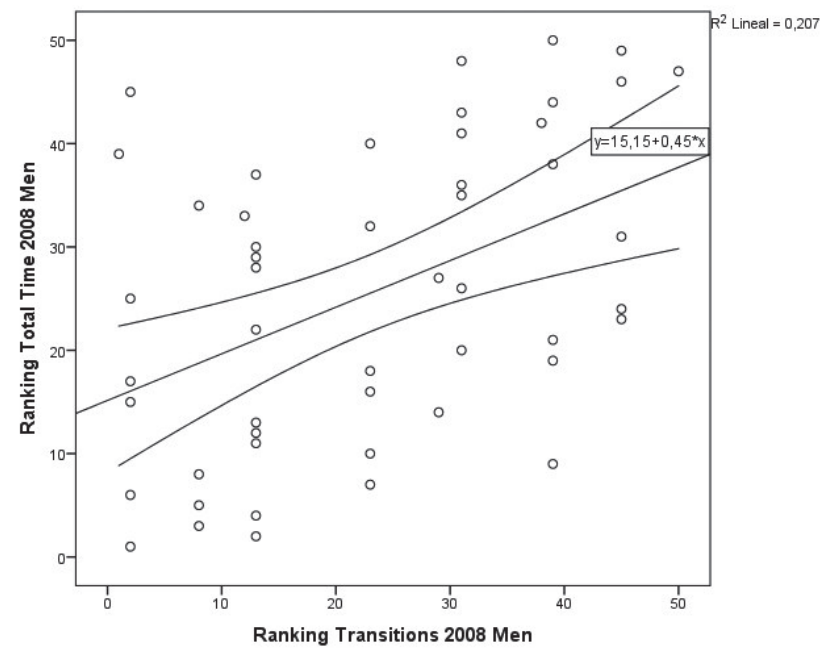

Figura 5. Relación entre ranking de la fase Transitions y ranking Total Time de la prueba.

Los rankings de las fases con el ranking del Total Time en el triatlón de los JJOO de Sídney 2000, (Fernández-Revelles, 2017), Atenas 2004 (Fernández-Revelles et al., 2018) y Beijing 2008 muestran resultados muy diferentes. Sin embargo, también podemos encontrar similitudes como es el tiempo empleado en cada fase. Así la fase Swim ha empleado 16,14\% en Sídney 2000, 15,70\% en Atenas 2004 y 16,40\% en Beijing 2008. La fase Bike ha empleado 53,15\% en Sídney 2000, $54,36 \%$ en Atenas 2004 y 52,55\% en Beijing 2008. La fase Run ha empleado 30,07\% en Sídney 2000, 29,41\% en Atenas 2004 y 30,21\% en Beijing 2008. La fase Transitions ha empleado 0,64\% en Sídney 2000, 0,53\% en Atenas 2004 y $0,84 \%$ en Beijing 2008. Lo podríamos resumir a que el tiempo empleado en las diferentes fases en los triatlones estudiados es aproximadamente 16\% Swim, 53\% Bike, 30\% Run, y el resto que no llega al $1 \%$ Transitions.

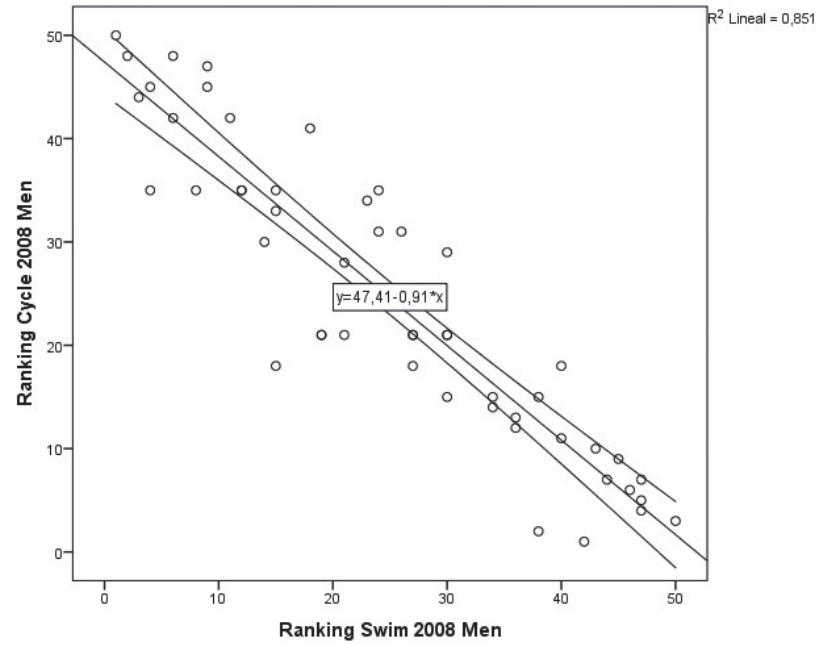

Figura 6. Relación entre ranking de la fase Swim y ranking de la fase Bike o Cycle

Analizando la relación de las correlaciones de los rankings entre fases encontramos que las fases Swim y Bike en Beijing 2008 (figura 6) tienen una relación inversamente proporcional con una correlación $r s=-0,931$; y $p=0,000$; lo que puede indicar que los atletas que se emplearon más en la fase Swim descansaron en la fase Bike. Resultado muy acentuado en Beijing 2008 y muy similar a Sídney 2000 con $r s=-0,480$; y $p=0,000$; pero muy diferente al resultado de Atenas 2004 con una correlación directamente proporcional, aunque baja de $r s=0,311$; y $\mathrm{p}=0,038$. 


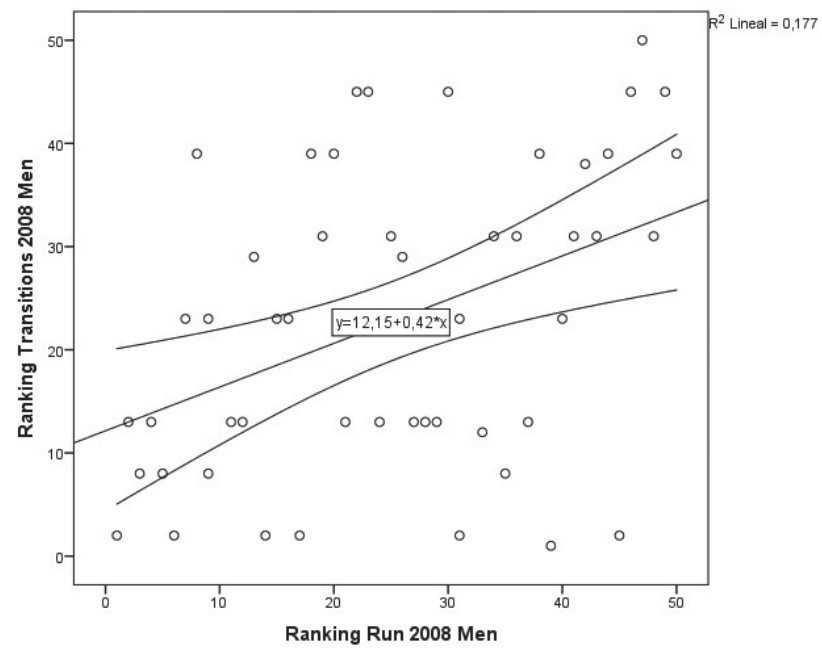

Figura 7. Relación entre ranking de la fase Run y ranking de la fase Transitions.

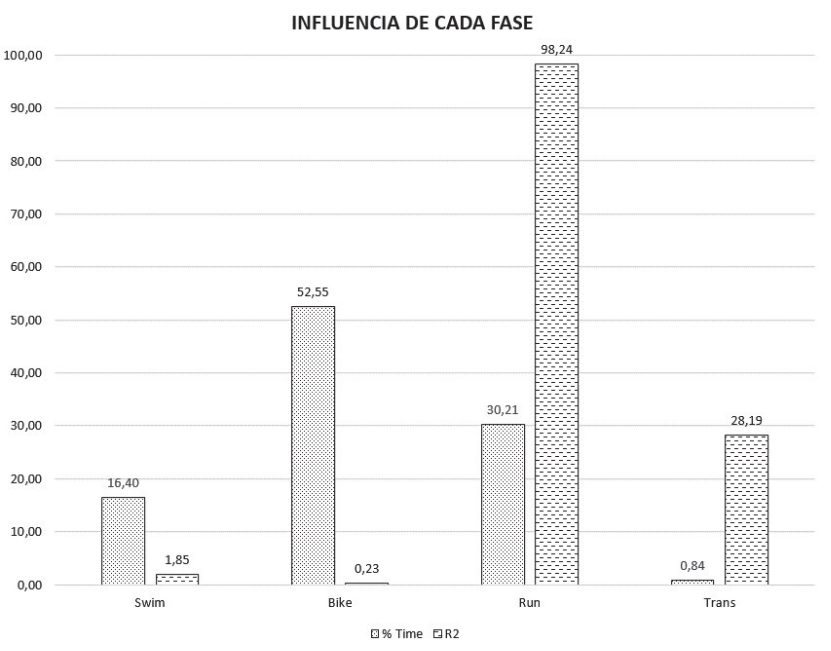

Figura 8. Influencia de cada fase en la prueba en función del porcentaje de tiempo empleado en cada fase y del porcentaje de ranking final determinado por cada fase en función del coeficiente de determinación lineal $R^{2}$.
Entre las demás fases de Beijing 2008 no existen correlaciones excepto la correlación moderada existente entre las fases Run y Transitions con $r s=0,409$; y $p=0,003$; (figura 7) resultado parecido a la correlación que tiene la fase Transitions con Total Time lo que puede significar una mayor predisposición en la preparación de las transiciones de aquellos que van luchar por la victoria final. Este resultado también es similar al encontrado en Sídney 2000 con $r s=0,391 ;$ y $p=0,006$.

\section{Conclusiones}

El resultado final del triatlón masculino en los JJOO de Beijing 2008 no viene determinado ni por el orden de las fases, ni por la duración de estas, y la fase menos influyente en el resultado final ha sido la fase Bike aun siendo la que más tiempo ha ocupado, y la fase más influyente ha sido la fase Run que es la última fase de la prueba.

Este tipo de estudios se fundamentado por la contraposición existente por la similitud de los tiempos empleados en las diferentes fases en los distintos triatlones y sin embargo la gran diferencia existente entre los rankings de las fases con el ranking Total Time.

En esta línea de trabajo habría que seguir estudiando los patrones que siguen estas pruebas en los JJOO, y continuar con otro tipo de triatlones en sus diferentes categorías, edades, distancias, sexo, etc...

Sería también interesante seguir esta línea de trabajo para ver qué conclusiones comunes se podrían extraer y que quizá pudieran determinar la modificación de algunas normas de competición o incluir factores correctores.

\section{Referencias}

1. Abbiss, C. R., \& Laursen, P. B. (2008). Describing and understanding pacing strategies during athletic competition. Sports Medicine, 38(3), 239-252.

2. Atkinson, G., \& Brunskill, A. (2000). Pacing strategies during a cycling time trial with simulated headwinds and tailwinds. Ergonomics, 43(10), 1449-1460. doi: 10.1080/001401300750003899

3. Bentley, D. J., Millet, G. P., Vleck, V. E., \& McNaughton, L. R. (2002). Specific aspects of contemporary triathlon - Implications for physiological analysis and performance. Sports Medicine, 32(6), 345-359. doi: 10.2165/00007256-200232060-00001

4. Bentley, D. J., Wilson, G. J., Davie, A. J., \& Zhou, S. (1998). Correlations between peak power output, muscular strength and cycle time trial performance in triathletes. Journal of Sports Medicine and Physical Fitness, 38(3), 201-207.
5. Bernard, T., Hausswirth, C., Le Meur, Y., Bignet, F., Dorel, S., \& Brisswalter, J. (2009). Distribution of Power Output during the Cycling Stage of a Triathlon World Cup. Medicine and Science in Sports and Exercise, 41(6), 1296-1302. doi: 10.1249/MSS.0b013e318195a233

6. Bonacci, J., Vleck, V., Saunders, P. U., Blanch, P., \& Vicenzino, B. (2013). Rating of perceived exertion during cycling is associated with subsequent running economy in triathletes. Journal of Science and Medicine in Sport, 16(1), 49-53. doi: 10.1016/j.jsams.2012.04.002

7. Dallam, G. M., Jonas, S., \& Miller, T. K. (2005). Medical considerations in triathlon competition - Recommendations for triathlon organisers, competitors and coaches. Sports Medicine, 35(2), 143-161. doi: 10.2165/00007256-200535020-00004

8. Etxebarria, N., D’Auria, S., Anson, J. M., Pyne, D. B., \& Fergu- 
son, R. A. (2014). Variability in Power Output During Cycling in International Olympic-Distance Triathlon. International Journal of Sports Physiology and Performance, 9(4), 732-734. doi: 10.1123/ ijspp.2013-0303

9. Fernandez-Revelles, A. B. (2012). ABFR-Index: correlación entre producción científica y Juegos Olímpicos 2008. Habilidad Motriz, 38, 51 57. doi: http://hdl.handle.net/10481/29518

10. Fernandez-Revelles, A. B. (2013). Modelo matemático de ley de potencias aplicado al maratón. Habilidad Motriz, 41, 12-20. doi: http://hdl. handle.net/10481/29518

11. Fernandez-Revelles, A. B. (2014). ABFR-Index: correlation between "soccer" scientific production and ranking. Revista Internacional De Medicina Y Ciencias De La Actividad Fisica Y Del Deporte, 14(56), 705-718. doi: Http://cdeporte.rediris.es/revista/revista56/artABFR506.htm

12. Fernandez-Revelles, A. B. (2017). Correlation between phases and final result in Men's triathlon competition at the Olympic Games in Sydney 2000. Retos-Nuevas Tendencias En Educacion Fisica Deporte Y Recreacion(32), 167-171. doi: http://recyt.fecyt.es/index.php/retos/article/ download/52952/33666

13. Fernandez-Revelles, A. B. (2018). Infographic. Correlation between phases and final result in Men's triathlon competition at the Olympic Games in Sydney 2000. British Journal of Sports Medicine. doi: 10.1136/ bjsports-2018-099225

14. Fernández-Revelles, A. B. (2017). Correlación en triatlón masculino entre fases y resultado final en los JJOO de Sídney 2000 / Men's triathlon correlation between the phases and the final result in the Olympic Games in Sydney 2000. Retos. Nuevas tendencias en Educación Física, Deporte y Recreación, 32, 167-171. doi: http://recyt.fecyt.es/index.php/ retos/article/download/52952/33666

15. Fernandez-Revelles, A. B., Espejo-Garcés, T., Ubago-Jiménez, J. L., \& Chacón-Cuberos, R. (2018). Men's triathlon correlation between the phases and the final result in the Olympic Games in Athens 2004. Journal of Sport and Health Research(IN-PRESS).

16. Fernandez-Revelles, A. B., Robles, A., Dafos, J., Soto, V. M., PerezCortes, A. J., Latorre, P., . . . Romero, C. (2009). Physical activity: Evaluation of research in Spain. Gaceta sanitaria, 23, 204-204.

17. Fernandez-Revelles, A. B., Ubago-Jiménez, J. L., Padial-Ruz, R., Castro-Sánchez, M., Viciana-Garófano, V., \& Puertas-Molero, P. (2018). Men's triathlon correlation between the phases and result in the Olympic Games in Beijing 2008. SPORT TK Revista Euroamericana de Ciencias del Deporte, $7(2)$.

18. Hausswirth, C., \& Brisswalter, J. (2008). Strategies for Improving Performance in Long Duration Events Olympic Distance Triathlon. Sports Medicine, 38(11), 881-891. doi: 10.2165/00007256200838110-00001

19. Hausswirth, C., Le Meur, Y., Bieuzen, F., Brisswalter, J., \& Bernard, T. (2010). Pacing strategy during the initial phase of the run in triathlon: influence on overall performance. European Journal of Applied Physiology, 108(6), 1115-1123. doi: 10.1007/s00421-0091322-0

20. Hue, O. (2011). The Challenge of Performing Aerobic Exercise in Tropical Environments: Applied Knowledge and Perspectives. International Journal of Sports Physiology and Performance, 6(4), 443-454.

21. ITU International Triathlon Union. (2017a). ITU Competition Results. Retrieved 9-09, 2016, from http://www.triathlon.org/results

22. ITU International Triathlon Union. (2017b, Mayo-2017). ITU Competition Rules. Retrieved 9-09, 2016, from http://www.triathlon.org/ about/documents

23. Jaenes Sánchez, J. C., Peñaloza Gómez, R., Navarrete Dueñas, K. G., \& Bohórquez Gómez-Millán, M. R. (2012). Ansiedad y autoconfianza precompetitiva en triatletas. Revista iberoamericana de psicología del ejercicio y el deporte, 7(1), 113-124.

24. Jeukendrup, A. E., Jentjens, R., \& Moseley, L. (2005). Nutritional considerations in triathlon. Sports Medicine, 35(2), 163-181. doi: 10.2165/00007256-200535020-00005

25. Johnson, E. C., Pryor, J. L., Casa, D. J., Belval, L. N., Vance, J. S. DeMartini, J. K., ... Armstrong, L. E. (2015). Bike and run pacing on downhill segments predict Ironman triathlon relative success. Journal of Science and Medicine in Sport, 18(1), 82-87. doi: 10.1016/j. jsams.2013.12.001

26. Kerr, C. G., Trappe, T. A., Starling, R. D., \& Trappe, S. W. (1998). Hyperthermia during Olympic triathlon: influence of body heat storage during the swimming stage. Medicine and Science in Sports and Exercise, 30(1), 99-104. doi: 10.1097/00005768-199801000-00014

27. Le Meur, Y., Bernard, T., Dorel, S., Abbiss, C. R., Honnorat, G., Brisswalter, J., \& Hausswirth, C. (2011). Relationships Between Triathlon Performance and Pacing Strategy During the Run in an International Competition. International Journal of Sports Physiology and Performance, 6(2), 183-194

28. Le Meur, Y., Hausswirth, C., Dorel, S., Bignet, F., Brisswalter, J., \& Bernard, T. (2009). Influence of gender on pacing adopted by elite triathletes during a competition. European Journal of Applied Physiology, 106(4), 535-545. doi: 10.1007/s00421-009-1043-4

29. Le Meur, Y., Hausswirth, C., Natta, F., Couturier, A., Bignet, F., \& Vidal, P. P. (2013). A multidisciplinary approach to overreaching detection in endurance trained athletes. Journal of Applied Physiology, 114(3), 411-420. doi: 10.1152/japplphysiol.01254.2012

30. Leruite, M., Morente-Sánchez, J., Martos, P., Girela, M. J., \& Zabala, M. (2016). Analysis of the Sporting Context of Spanish Female Competitive Cyclists and Triathletes. Revista Internacional De Medicina $Y$ Ciencias De La Actividad Fisica Y Del Deporte, 16(64), 667-684.

31. Méndez-Giménez, A., \& Fernández-Río, J. (2011). Análisis y modificación de los juegos y deportes tradicionales para su adecuada aplicación en el ámbito educativo. Retos. Nuevas tendencias en Educación Física, Deporte y Recreación, 19, 54-58.

32. Millet, G. P., \& Vleck, V. E. (2000). Physiological and biomechanical adaptations to the cycle to run transition in Olympic triathlon: review and practical recommendations for training. British Journal of Sports Medicine, 34(5), 384-390. doi: 10.1136/bjsm.34.5.384

33. Nacke, L. E., Bateman, C., \& Mandryk, R. L. (2011). BrainHex: Preliminary Results from a Neurobiological Gamer Typology Survey. In J. Anacleto, S. Fels, N. Graham, B. Kaparalos, M. S. ElNasr, \& K. Stanley (Eds.), Entertainment Computing - Icec 2011 (Vol. 6972, pp. 288-293). Berlin: Springer-Verlag Berlin.

34. Noakes, T. D. (2007). Drinking guidelines for exercise: What evidence is there that athletes should drink "as much as tolerable", "to replace the weight lost during exercise" or "ad libitum"? Journal of Sports Sciences, 25(7), 781-796. doi: 10.1080/02640410600875036

35. Peeling, P., \& Landers, G. (2009). Swimming intensity during triathlon: A review of current research and strategies to enhance race performance. Journal of Sports Sciences, 27(10), 1079-1085. doi: 10.1080/02640410903081878

36. Ramos-Campo, D. J., Martínez, F., Esteban, P., Rubio-Arias, J. A., \& Jiménez, J. F. (2016). Intermittent hypoxic training and cycling performance in triathletes. Revista Internacional De Medicina Y Ciencias De La Actividad Fisica Y Del Deporte, 16(61), 139-156. doi: http://dx.doi. org/10.15366/rimcafd2016.61.011

37. Rivas, L. G., Mielgo-Ayuso, J., Norte-Navarro, A., Cejuela, R., Cabanas, M. D., \& Martinez-Sanz, J. M. (2015). Body composition and somatotype in university triathletes. Nutricion Hospitalaria, 32(2), 799 807. doi: 10.3305/nh.2015.32.2.9142

38. Tondello, G. F., Wehbe, R. R., Diamond, L., Busch, M., Marczewski, A., Nacke, L. E., \& Acm. (2016). The Gamification User Types Hexad Scale. Chi Play 2016: Proceedings of the 2016 Annual Symposium on Computer-Human Interaction in Play, 229-243. doi: $10.1145 / 2967934.2968082$ 
39. Zapico, A. G., Benito, P. J., Diaz, V., Ruiz, J. R., \& Calderon, F. J. (2014). Heart rate profile in highly trained triathletes. Revista Internacional De Medicina Y Ciencias De La Actividad Fisica Y Del Deporte,
14(56), 619-632. doi: Http://cdeporte.rediris.es/revista/revista56/artperfil $505 . h \mathrm{tm}$ 